\title{
Knockdown of autophagy-related gene BECLIN1 promotes cell growth and inhibits apoptosis in the A549 human lung cancer cell line
}

\author{
WENYU WANG ${ }^{1,2}$, HONGKUN FAN $^{3}$, YUN ZHOU $^{2}$, PING DUAN $^{3}$, GUOQIANG ZHAO $^{3}$ and GANG WU ${ }^{1}$ \\ ${ }^{1}$ Cancer Center, Union Hospital, Tongji Medical College, Huazhong University of Science and Technology, Wuhan, \\ Hubei 430023; ${ }^{2}$ Department of Medical Oncology, Henan Province People's Hospital, Zhengzhou, Henan 450003; \\ ${ }^{3}$ College of Basic Medical Science, Zhengzhou University, Zhengzhou, Henan 450001, P.R. China
}

Received December 14, 2012; Accepted March 7, 2013

DOI: $10.3892 / \mathrm{mmr} .2013 .1379$

\begin{abstract}
The expression of BECLIN1 is significantly reduced in non-small cell lung cancer (NSCLC) compared with non-cancerous tissue. However, the role of BECLIN1 in lung cancer is unclear. Using the RNA interference (RNAi) technique the present study investigated the effect of the knockdown of BECLIN1 on the cell growth and proliferation of the A549 human lung cancer cell line. The target site for the RNAi technique was designed and the lentivirus vector for the small interfering (si)RNA expression was constructed according to the encoding sequence of the mRNA for BECLIN1. The A549 cells were transfected with the siRNA virus against BECLIN1. BECLIN1 expression was detected by reverse transcription (RT)-PCR and western blot analysis. The 3-(4,5-dimethylthiazol-2-yl)-2,5-diphenyltetrazolium bromide (MTT) method was applied to detect cell growth. Flow cytometry was used to determine cell apoptosis. The activity of caspase- 3 and caspase- 9 was also detected in the A549 cells with BECLIN1 knockdown. The results showed that siRNA virus transfection significantly decreased the mRNA and protein expression of BECLIN1 in the transfected A549 cells. The knockdown of BECLIN1 promoted cell growth and decreased apoptosis. Caspase-3 and -9 activity in the A549 cells with BECLIN1 knockdown was significantly reduced. In conclusion, the siRNA expression vector effectively inhibited the expression of BECLIN1 in the A549 human lung cancer cell line and promote the growth and proliferation of the A549 cells.
\end{abstract}

Correspondence to: Dr Gang Wu, Cancer Center, Tongji Union Hospital, Hangkong Road, 1277 Liberation Avenue, Wuhan, Hubei 430023, P.R. China

E-mail:wangwy0712@163.com

Key words: RNA interference, autophagy, BECLIN1, apoptosis, caspase

\section{Introduction}

Autophagy is the major cellular pathway for the degradation of long-lived proteins and cytoplasmic organelles (1). Autophagy is becoming an important area in cancer research $(2,3)$. Abnormal autophagy has certain associations with the formation of tumors $(4,5)$, however, autophagy in lung cancer has not yet been studied.

BECLIN-1 (the mammalian counterpart of the yeast Atg6 gene), which is part of a type III phosphatidylinositol 3-kinase complex, is required for autophagic vesicle formation $(6,7)$. BECLIN1 suppresses tumor formation via the lysosomal degradation pathway (8). BECLIN1 is monoallelically deleted in $\sim 75 \%$ of ovarian cancers $(9,10), 50 \%$ of breast cancers $(11)$ and $40 \%$ of prostate cancers (12). The expression of BECLIN1 has been observed to be significantly reduced in non-small cell lung cancer (NSCLC) compared with that in non-cancerous tissue (13), however, the role of BECLIN1 in lung cancer is unclear. In the present study, BECLIN1 was knocked down in the A549 human lung cancer cell line and its role in cell proliferation and apoptosis was studied.

\section{Materials and methods}

Main reagents. The lentiviral small interfering (si)RNA pRNAT-U6.2/Lenti vectors were obtained from GenScript Biotechnology (Piscataway, NJ, USA). The lentiviral packaging cell line 293T and the human lung cancer cell line A549 were purchased from the China Center for Type Culture Collection (CCTCC; Wuhan, Hubei, China). The E. coli DH5a restriction enzymes (BamHI and XhoI) and the T4 DNA ligase were purchased from Promega (Madison, WI, USA). The RPMI-1640 medium and the fetal bovine serum (FBS) were purchased from Hyclone Biotechnology (Waltham, MA, USA). The liposomes (Lipofectamine ${ }^{\mathrm{TM}}$ 2000) and G418 were purchased from Invitrogen Biotechnology (Carlsbad, CA, USA). The RNA Extraction, Plasmid DNA Extraction and Gel DNA Isolation kits were obtained from Qiagen Biotechnology (Hilden, Germany). The study was approved by the ethics committee of Zhengzhou University. 
Construction of the siRNA plasmid against BECLIN1. Promega, Ambion and Invitrogen siRNA target sequence analysis and design software were used to scan the human-specific BECLIN1 cDNA coding sequences (NM_003766). The siRNA target sequences were based on basic design principles and a NCBI Basic Local Alignment Search Tool (BLAST) homology analysis. The target sequences were ascertained in 356-374 bits (GAGAGGAGCCATTTATTGA). The small hairpin (sh)DNA single-chains were synthesized and BamHI and $X h o I$ enzyme residues were added at the two ends. This process was performed by Shanghai Bio-engineering Biotechnology (Shanghai, China). The designed sequences encoding the region of BECLIN1 were inserted between the BamHI and XhoI sites of the pRNAT-U6.2/Lenti plasmid. Competent E.coli were transformed with the recombinants following the manufacturer's recommendations. The positive clones were confirmed by PCR amplification screening and sequencing.

siRNA transfection. Three recombinant lentiviral vectors, empty lentiviral vectors and secondary packaging plasmids were co-transfected into the $293 \mathrm{~T}$ cells using $36 \mathrm{ml}$ Lipofectamine 2000 reagent, according to the manufacturer's instructions. The obtained BECN1 siRNA lentiviral fake virus particle solutions were designated as Lv-si356 and the $\mathrm{Lv}$-control and were stored at $-80^{\circ} \mathrm{C}$ until use. The human NSCLC A549 cell lines were cultured in RPMI-1640 medium supplemented with $10 \%$ defined FBS. The day prior to infection, the cells were plated into a tissue culture plate to $80 \%$ confluence. The human NSCLC A549 cell lines were infected by the Lv-si356- and Lv-Control-prepared lentiviral particles. After $24 \mathrm{~h}$, the medium was replaced and the culture was continued. To obtain a stable infection, the cell lines were screened by G418 for 3 weeks.

RNA isolation and cDNA synthesis. Total RNA was isolated using TRIzol reagent (Invitrogen) following the manufacturer's instructions. The amount of RNA was measured spectrophotometrically by absorbance at $260 \mathrm{~nm}$ and the purity of the RNA was estimated by the ratio of the absorbance at A260/280. Reverse transcription of $2 \mu \mathrm{g}$ total RNA was performed with Moloney murine leukemia virus (MMLV) reverse transcriptase (Promega, USA) using oligo (dT) $)_{18}$ (Promega) as the reverse transcription primer. The reaction conditions for the reverse transcription were as follows: $2 \mu \mathrm{g}$ of total RNA was incubated with $100 \mathrm{pmol} / \mathrm{l}$ oligo $(\mathrm{dT})_{18}$ primer at $72^{\circ} \mathrm{C}$ for $10 \mathrm{~min}$ and rapidly chilled on ice, then $2 \mu 110 \mathrm{mM}$ dNTPs (Promega) and $4 \mu 1$ XX MMLV buffer (Promega) were added. The mixtures were incubated at $37^{\circ} \mathrm{C}$ for $5 \mathrm{~min}$. The mixture containing 200 units Moloney murine leukemia virus reverse transcriptase (Promega) was run at $42^{\circ} \mathrm{C}$ for $1 \mathrm{~h}$. The reaction was then heated at $72^{\circ} \mathrm{C}$ for $10 \mathrm{~min}$ and stored at $-20^{\circ} \mathrm{C}$ until use.

Quantitative real-time reverse transcription (RT)-PCR. To quantitatively determine the level of the mRNA expression of BECLIN1, the primer pairs were tested empirically for amplification from $100 \mathrm{ng}$ cDNA. The optimal annealing temperature was determined by testing the generation of a single band of the primers on the gels and then a specific primer was successfully identified for BECLIN1. Quantitative real-time PCR reactions were performed on the ABI Prism 7000 Sequence Detection System (Applied Biosystems, Foster City, CA, USA). For the SYBR-Green II-based quantitative real-time PCR reactions (Takara, Shiga, Japan), each $30 \mu 1$ reaction contained $0.4 \mu \mathrm{M}$ primer pairs, $100 \mathrm{ng}$ cDNA, $15 \mu 1$ SYBR-Green, $0.6 \mu 1$ ROX as a fluorescence internal control and $\mathrm{ddH}_{2} \mathrm{O}$. The primers were as follows: BECLIN1 forward, 5'-GGCTGAGAGACTGGATCAGG-3' and reverse, 5'-CTGCGTCTGGGCATAACG-3'; GAPDH forward, 5'-GGACTGACCTGCCGTCTAG-3' and reverse, 5'-TAGCCCAGGATGCCCTTGAG-3'. The amplification program comprised two stages, with an initial $95^{\circ} \mathrm{C}$ Taq activation stage for $10 \mathrm{~min}$ followed by 45 cycles of $95^{\circ} \mathrm{C}$ denaturation for $5 \mathrm{sec}$ and $60^{\circ} \mathrm{C}$ annealing for $35 \mathrm{sec}$. Subsequent to the amplification, a melting curve analysis was performed by collecting fluorescence data. GAPDH served as an internal control. The comparative threshold cycle $\left(2^{-\Delta \Delta C T}\right)$ method was used to enable quantification of the mRNA of these genes. All sample analyses were performed in duplicate. The relative amount of the target gene was calculated using the $2^{-\triangle \Delta C T}$ method. The relative amplification efficiencies of the primers were tested and shown to be similar.

Western blotting. The uninfected A549 cells and the four groups of infected cells were added into the RLT buffer. Each group of cells was harvested and the protein concentrations were determined with a Bicinchoninic Acid (BCA) Protein Assay kit. The samples were dissolved in loading buffer, boiled for $5 \mathrm{~min}$ and then loaded onto $10 \%$ sodium dodecyl sulfate-polyacrylamide gel electrophoresis (SDS-PAGE). The protein bands were transferred onto a polyvinylidene difluoride membrane, which was blocked overnight at $4^{\circ} \mathrm{C}$ by Tris-buffered saline with Tween 20 (TBST) containing 5\% skimmed milk. The blocked membranes were then incubated with rat anti-human BECLIN1 antibodies (1:150) for $2 \mathrm{~h}$. The blocked membranes were then washed three times and incubated with horseradish peroxidase-conjugated rabbit anti-rat antibodies (1:2000) for $1 \mathrm{~h}$. The bands were visualized using 3,3'-diaminobenzidine (DAB).

3-(4,5-dimethylthiazol-2-yl)-2,5-diphenyltetrazolium bromide (MTT) method. The cells were seeded onto 96-well plates at a density of $5 \times 10^{4}$ cells per well in $100 \mu 1$ medium. All the cells were maintained in a humidified incubator at $37^{\circ} \mathrm{C}$ with $5 \% \mathrm{CO}_{2}$. A total of $20 \mu \mathrm{l}$ MTT (5 g/l) was added to each well and the absorbance at $570 \mathrm{~nm}$ was measured by a microplate reader. Subsequent to $4 \mathrm{~h}$ incubation, the number of surviving cells was measured. There were 5 parallel wells for each group.

Detection of apoptosis by flow cytometry. Subsequent to 48-h incubation, each group of cells was digested by trypsin. The cells were further incubated at a density of $5 \times 10^{5}-1 \times 10^{6} / \mathrm{ml}$ nutrient solution containing $75 \%$ cold ethanol overnight. Subsequent to centrifugation, $1 \mathrm{ml}$ of the cells were fixed with $100 \mu 1 \mathrm{RNA}$ enzymes $(1 \mathrm{mg} / \mathrm{ml})$ at $37^{\circ} \mathrm{C}$ for $30 \mathrm{~min}$. Then $100 \mu 110 \mu \mathrm{g} / \mathrm{ml}$ propidium iodide (PI) dye liquor was added in the dark. Following fixation, the cells were incubated in the dark for $30 \mathrm{~min}$. The samples were filtered through a 300 mesh nylon net, then the cell apoptosis rates were detected by flow cytometry. 
A

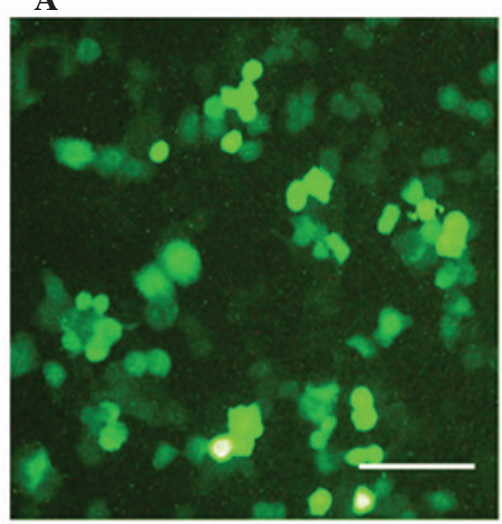

B

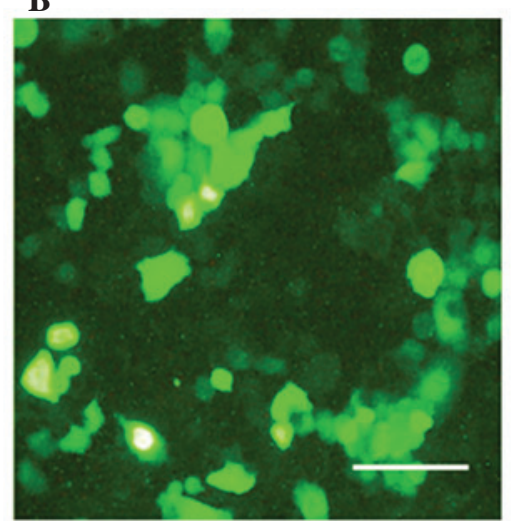

C

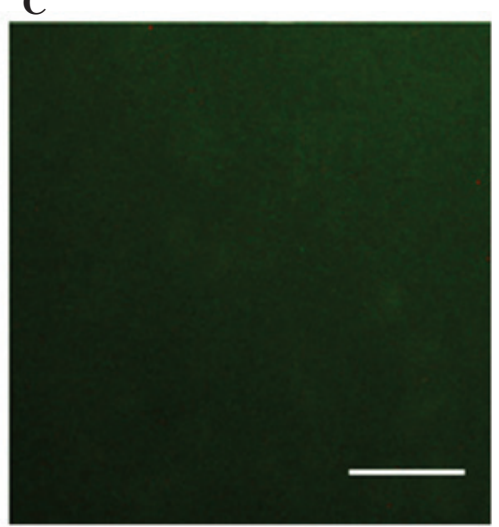

Figure 1. A549 cells transfected with lentivirus for $48 \mathrm{~h}$ shown using fluorescence microscopy. A549 cells transfected with (A) siRNA virus or (B) scramble virus. In total, $>90 \%$ of the cells were EGFP-positive. (C) Normal A549 cells. Scale, $200 \mu$ m. EGFP, enhanced green fluorescent protein.

A

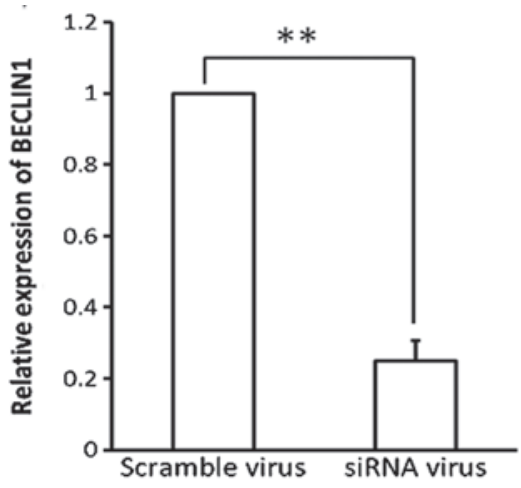

B

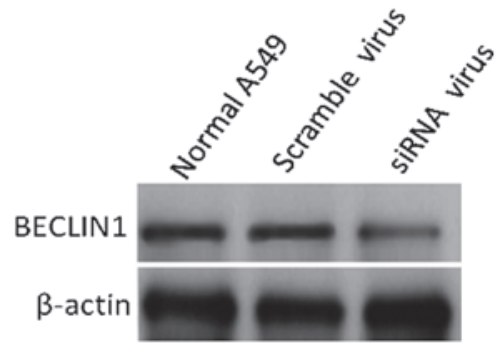

Figure 2. siRNA knockdown of BECLIN1 expression in the A549 cells. (A) RT-PCR and (B) western blot results of BECLIN1 expression. Small interfering (si)RNA virus transfection decreased the BECLIN1 expression. The mean and SEM of three independent A549 cell cultures are shown. ${ }^{* *}$ P $<0.01$. RT-PCR, reverse transcription PCR.

Detection of active caspase-3 and caspase-9. Each group of $\sim 5 \times 10^{5}$ cells was centrifuged at $500 \mathrm{x} \mathrm{g}$ for $5 \mathrm{~min}$. The collected cells were fixed into ice cold cell lysate at a density of $50 \mu \mathrm{l} / 2 \times 10^{5}$ cells for $10 \mathrm{~min}$. The microtubes were centrifuged at 20,000 x g for $5 \mathrm{~min}$, maintaining a consistent temperature of $4^{\circ} \mathrm{C}$. Thereafter, the supernatants were transferred to another set of ice-cold microtubes. The $50 \mu 1$ samples were pipetted into a 96-well plate, mixed and sealed with paraffin wax. Subsequent to a 2-h incubation in the dark, the fluorescence was determined by a plate reader using an excitation wavelength of $380 \mathrm{~nm}$ and an emission wavelength of $460 \mathrm{~nm}$.

Statistical analysis. Statistical analyses were performed using SPSS 13.0 (SPSS, Chicago, IL, USA). Quantitative data are presented as the mean \pm standard deviation. Comparisons among all the groups were performed with a one-way analysis of variance (ANOVA) test. Comparisons between two groups were evaluated with a t-test.

\section{Results}

Lentivirus-mediated siRNA vector transfection in the A549 human lung cancer cell line. Following transfection with the siRNA virus against BECLIN1 for $48 \mathrm{~h}$, the A549 cells expressed enhanced green fluorescent protein (EGFP). In total, $>90 \%$ of the cells were EGFP-positive in the cell culture (Fig. 1). Subsequent to 2 weeks of G418 selection, the BECLIN1 knockdown A549 cell line was established.

Decrease of BECLIN1 expression following siRNA virus transfection in the A549 cells. Subsequent to transfection with the siRNA and scramble viruses for $48 \mathrm{~h}$, BECLIN1 expression was detected using RT-PCR and western blotting (Fig. 2). The mRNA and protein expression of BECLIN1 decreased in the siRNA virus-transfected A549 cells.

Knockdown of BECLIN1 increases A549 cell proliferation. Cell proliferation was detected by MTT assay in the A549 cells transfected with the siRNA or scramble viruses. Normal A549 cells were assayed as the blank control. The knockdown of BECLIN1 increased cell proliferation significantly (Fig. 3).

Knockdown of BECLIN1 inhibits apoptosis in the A549 cells. Apoptosis was detected by PI staining and flow cytometry in the A549 cells transfected with the siRNA or scramble viruses. Normal A549 cells were assayed as the blank control. The apoptosis index (AI) was less in the BECLIN1-knockdown A549 cells than that in the scramble virus-transfected A549 cells and blank controls $(\mathrm{P}<0.05$; Fig. 4$)$. 


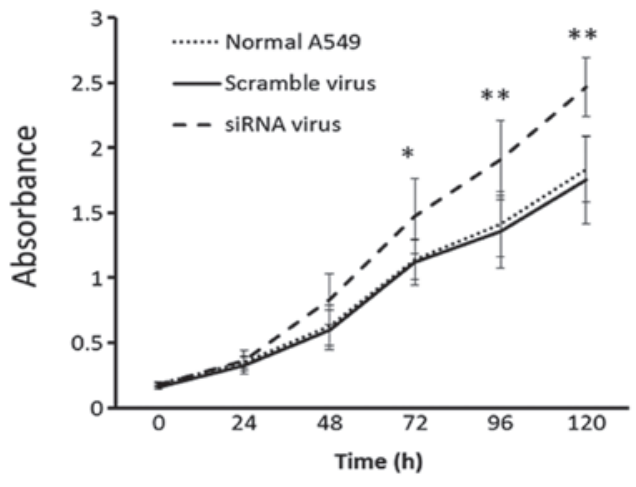

Figure 3. MTT assay results. The knockdown of BECLIN1 increased the cell proliferation significantly. The data represent the mean and SEM of three independent A549 cell cultures. ${ }^{* *} \mathrm{P}<0.01 ;{ }^{*} \mathrm{P}<0.05$. MTT, 3-(4,5-dimethylthiazol-2-yl)-2,5-diphenyltetrazolium bromide; siRNA, small interfering RNA.
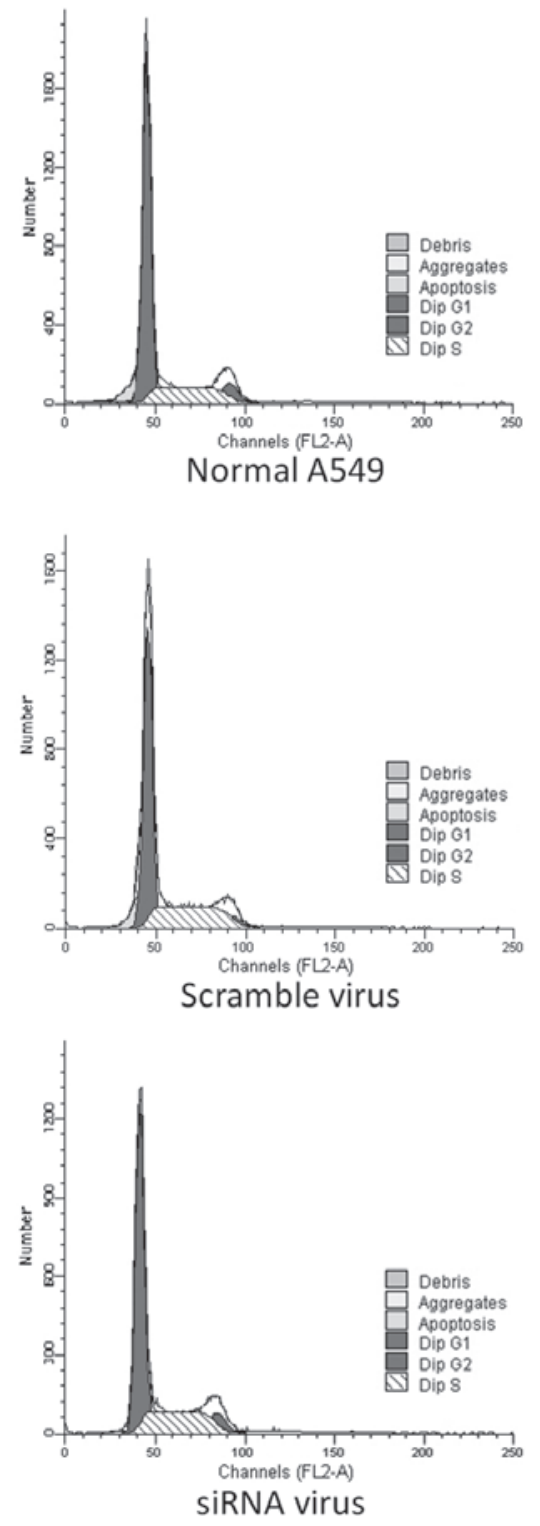

Figure 4. Detection of apoptosis by flow cytometry. The knockdown of BECLIN1 decreased apoptosis in A549 cells. The apoptosis index (AI) was $4.31 \pm 0.49 \%$ in the BECLIN1-knockdown A549 cells, which was less than the PI value in the scramble virus-transfected A549 $(8.29 \pm 1.12 \%)$ and normal A549 $(9.12 \pm 1.21 \%$; $\mathrm{P}<0.05)$ cells. The apoptosis analyses are representative of three independent experiments. siRNA, small interfering RNA.

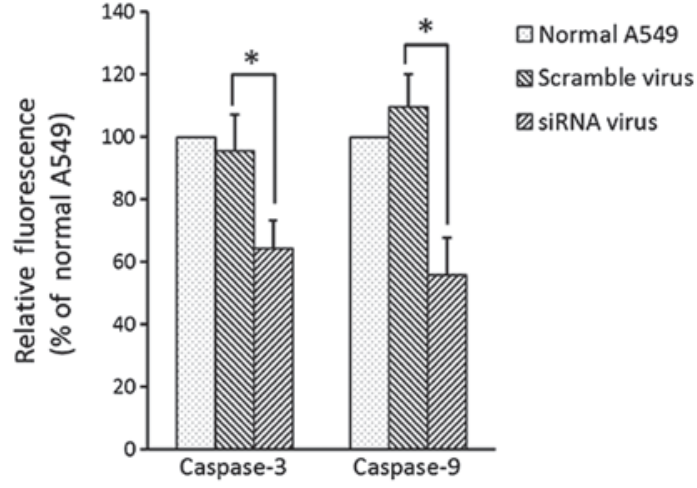

Figure 5. Detection of caspase-3 and caspase-9 activity in the A549 cells. The activity of caspase- 3 and caspase- 9 decreased in the A549 cells following siRNA transfection in contrast to the activity in the normal A549 cells and the A549 cells following scramble transfection. The data show the mean and SEM of three independent A549 cell cultures. ${ }^{*} \mathrm{P}<0.05$. siRNA, small interfering RNA.

Knockdown of BECLIN1 inhibits the activity of caspase-3 and caspase-9. The activity of caspase- 3 and caspase- 9 decreased in the A549 cells following siRNA transfection in contrast with the activity in the normal A549 cells and the A549 cells following scramble transfection (Fig. 5). There was no difference in the activity of caspase- 3 and caspase- 9 between the normal A549 and scramble virus-transfected A549 cells.

\section{Discussion}

The role of autophagy in tumorigenesis is controversial. Autophagy inhibitors (chloroquine) and autophagy promoters (rapamycin) block tumorigenesis by an unknown mechanism(s) $(14,15)$. This is known as the "Autophagy Paradox'. Little is known about the role of autophagy in the tumorigenesis of NSCLC.

BECLIN1 is a key regulator of autophagy (16) and is involved in cell proliferation and tumor formation. BECLIN1 triggers autophagy in human breast carcinoma cells: this autophagy-promoting activity was observed to be associated with the inhibition of cell proliferation in vitro and with tumorigenesis in nude mice (17). Exogenous BECLIN1 expression in MCF-7 breast cancer cells inhibits the cell proliferation and tumorigenesis of these cells (17). In BECLIN1-knockout transgenic mice, the incidence of lung adenocarcinoma, liver cancer and lymphoma increases significantly $(18,19)$. The expression of BECLIN1 is significantly decreased in lung cancer tissues, suggesting that autophagy may be involved in the pathogenesis of lung cancer (20). In the present study, BECLIN1 knockdown increased cell proliferation in the A549 cells. This suggested that BECLIN1 expression may be a significant factor in maintaining the normal level of cell proliferation and that the downregulation or depletion of BECLIN1 in lung cancer may be an etiology responsible for the increased cell proliferation in cancer.

Certain studies have demonstrated the paradoxical correlation between BECLIN1 and apoptosis. Liang et al (17) showed that the BECLIN1 gene reduced apoptosis of the central nervous system. Wang (7) demonstrated that the depletion of BECLIN1 activated apoptosis. However, BECLIN1 was also 
suggested to bind the Bcl-2 family members, which may have a direct role in initiating apoptotic signaling $(21,22)$. In the present study, the inhibition of apoptosis and the decrease in caspase- 3 and caspase- 9 activity by BECLIN1 knockdown was validated in the A549 cells. It appears that BECLIN1 may play a different role in the regulation of apoptosis, which therefore requires further elucidation. When considering the promotive effect of BECLIN1 in the apoptosis of the A549 cells, we considered the theory that BECLIN1 downregulation may aid lung cancer cells to escape the body's normal clearance mechanism by apoptosis inhibition. This would contribute to the development of malignant tumors.

Taking these results together, BECLIN1 knockdown increases cell proliferation and decreases apoptosis in lung cancer cells. Therefore, BECLIN1 may be a biological target for gene therapy and the upregulation of BECLIN1 may be a promising treatment method for lung cancer, or even other tumor cells. This may provide further insight into the treatment of cancer.

\section{Acknowledgements}

The authors would like to express their particular appreciation to Dr Yongmin Liu who provided statistical assistance.

\section{References}

1. Kanzawa T, Germano IM, Komata T, Ito H, Kondo Y and Kondo S: Role of autophagy in temozolomide-induced cytotoxicity for malignant glioma cells. Cell Death Differ 11: 448-457, 2004.

2. Carew JS, Kelly KR and Nawrocki ST: Autophagy as a target for cancer therapy: new developments. Cancer Manag Res 4 357-365, 2012

3. Meschini S, Condello M, Lista P and Arancia G: Autophagy: molecular mechanisms and their implications for anticancer therapies. Curr Cancer Drug Targets 11: 357-379, 2011.

4. Yang ZJ, Chee CE, Huang S and Sinicrope FA: The role of autophagy in cancer: therapeutic implications. Mol Cancer Ther 10: 1533-1541, 2011

5. Lockshin RA and Zakeri Z: Caspase-independent cell deaths. Curr Opin Cell Biol 14: 727-733, 2002.

6. Sun Q, Fan W and Zhong Q: Regulation of Beclin 1 in autophagy. Autophagy 5: 713-716, 2009.
7. Wang J: Beclin 1 bridges autophagy, apoptosis and differentiation. Autophagy 4: 947-948, 2008.

8. Shintani T and Klionsky DJ: Autophagy in health and disease: a double-edged sword. Science 306: 990-995, 2004.

9. Ibragimova I and Cairns P: Assays for hypermethylation of the BRCA1 gene promoter in tumor cells to predict sensitivity to PARP-inhibitor therapy. Methods Mol Biol 780: 277-291, 2011.

10. Tangir J, Muto MG, Berkowitz RS, Welch WR, Bell DA and Mok SC: A $400 \mathrm{~kb}$ novel deletion unit centromeric to the BRCA1 gene in sporadic epithelial ovarian cancer. Oncogene 12: 735-740, 1996.

11. Saito H, Inazawa J, Saito S, et al: Detailed deletion mapping of chromosome 17q in ovarian and breast cancers: 2 -cM region on $17 \mathrm{q} 21.3$ often and commonly deleted in tumors. Cancer Res 53: 3382-3385, 1993.

12. Gao X,Zacharek A, Salkowski A, et al: Loss of heterozygosity of the BRCA1 and other loci on chromosome 17q in human prostate cancer. Cancer Res 55: 1002-1005, 1995.

13. Liu Q, Wang JJ, Pan YC, Meng LF, Zhan X and Zheng QF: Expression of autophagy-related genes Beclin1 and MAPLC3 in non-small cell lung cancer. Ai Zheng 27: 25-29, 2008 (In Chinese).

14. Martinez-Outschoorn UE, Whitaker-Menezes D, Pavlides S, et al: The autophagic tumor stroma model of cancer or 'battery-operated tumor growth': a simple solution to the autophagy paradox. Cell Cycle 9: 4297-4306, 2010.

15. Maycotte P, Aryal S, Cummings CT, Thorburn J, Morgan MJ and Thorburn A: Chloroquine sensitizes breast cancer cells to chemotherapy independent of autophagy. Autophagy 8: 200-212, 2012.

16. Ruck A, Attonito J, Garces KT, et al: The Atg6/Vps30/Beclin 1 ortholog BEC-1 mediates endocytic retrograde transport in addition to autophagy in C.elegans. Autophagy 7: 386-400, 2011

17. Liang XH, Jackson S, Seaman M, et al: Induction of autophagy and inhibition of tumorigenesis by beclin 1 . Nature 402: 672-676, 1999.

18. Lozy F and Karantza V: Autophagy and cancer cell metabolism. Semin Cell Dev Biol 23: 395-401, 2012.

19. Yue Z, Jin S, Yang C, Levine AJ and Heintz N: Beclin 1, an autophagy gene essential for early embryonic development, is a haploinsufficient tumor suppressor. Proc Natl Acad Sci USA 100: 15077-15082, 2003.

20. Jiang ZF, Shao LJ, Wang WM, Yan XB and Liu RY: Decreased expression of Beclin-1 and LC3 in human lung cancer. Mol Biol Rep 39: 259-267, 2012.

21. Oberstein A, Jeffrey PD and Shi Y: Crystal structure of the Bcl-XL-Beclin 1 peptide complex: Beclin 1 is a novel BH3-only protein. J Biol Chem 282: 13123-13132, 2007.

22. Maiuri MC, Le Toumelin G, Criollo A, et al: Functional and physical interaction between $\mathrm{Bcl}-\mathrm{X}(\mathrm{L})$ and a BH3-like domain in Beclin-1. EMBO J 26: 2527-2539, 2007. 